\title{
LE DISCOURS SUR LA GUERRE D'ESPAGNE DANS L'ESPOIR D'ANDRÉ MALRAUX ${ }^{1}$
}

\author{
MAMADOU ABDOULAYE LY \\ Yunnan Normal University
}

\begin{abstract}
Résumé
La guerre d'Espagne occupe une importance capitale dans la vie et l'œuvre d'André Malraux. En premier lieu, Malraux s'engage comme aviateur dans les brigades internationales de soutien aux Républicains espagnols. En second lieu, il consacre plusieurs textes à la guerre d'Espagne dans La Tête d'obsidienne et dans Hôtes de passage. Mais c'est L'espoir qui cristallise le travail d'écrivain de Malraux sur l'Espagne car cette oeuvre n'est pas seulement un reportage sur cette guerre. Ce texte constitue plutôt un poème dédié à la révolution espagnole. Ce travail d'écriture passe essentiellement par le discours que Malraux développe dans le tissu romanesque à travers la parole des personnages et la voix du narrateur. Ainsi, Malraux passe de la discussion entre Alvear et Scali sur le dilemme entre l'action révolutionnaire et le culte de l'art à l'éclatement de la voix du récit entre les chefs révolutionnaires et à l'intervention de l'auteur pour approfondir le débat sur la question de la Révolution et de l'art de la guerre.
\end{abstract}

Mots-clés: Guerre d’Espagne, Espoir, Discours, Focalisation, Voix off.

\begin{abstract}
The war of Spain occupies a central importance in the life and work of Andre Malraux. Firstable, Malraux enters as a pilot in the international brigades of the Spanish Republicans support. Second, he devoted several texts to the war of Spain in La Tête d'obsidienne and Hôtes de passage. But it is The Hope that crystallizes Malraux's works on Spain because this work is not only a report on this war. This text is rather a dedicated poem to the Spanish revolution. This kind of writing essentially passes by the speech that Malraux develops in fiction tissue through the word of the characters and the voice of the Narrator. Thus, Malraux goes from Alvear's and Scali's discussion on the dilemma between the revolutionary action and the cult of art to the exploding of the voice of the story between fighter's heads and the intervention of the author to deepen the debate on the issue of Revolution and war.
\end{abstract}

Key words: Spanish civil war, Hope, Speech, Focus, Off voice.

\section{INTRODUCTION}

Les rapports de Malraux avec l'Espagne présentent plusieurs visages. D'abord Malraux s'engage dans la défense de la cause républicaine aux côtés des Républicains

1 Yunnan Normal University. Correo: malysn@yahoo.fr. Recibido: 20-12-2011. Aceptado: 24-02-2012. 
espagnols dans les années 1936-1937 et crée 1’Escadrille Espagne. Ensuite, il effectue une tournée de conférences aux Etats-Unis pour récolter des fonds pour soutenir les troupes républicaines qui combattent l'armée de Franco. A cette intense activité militante en faveur des Républicains espagnols, s'ajoute l'écriture en 1937 du roman L'espoir consacré à cette guerre d'Espagne. Ce roman marque, après Le Temps du mépris en 1935, la seconde traduction littéraire du combat antifasciste de Malraux en Europe. Ce roman sera suivi par le tournage du film Espoir, sierra de Teruel en 1938, film qui ne sera projeté qu'en 1945. Il existe aussi un texte que de nombreux critiques considèrent une suite de L'espoir qui sera publiée dans le tome II des CEuvres complètes de Malraux en 1996 sous le titre de «Et sur la terre...».

L'engagement de Malraux dans la guerre d'Espagne nourrit donc son écriture romanesque et mémoriale car il évoquera cette période de sa vie dans La Tête d'obsidienne consacrée à Pablo Picasso en 1974 et dans Hôtes de passage grâce au personnage imaginaire Méry, présenté comme un ancien compagnon de Malraux durant la guerre d'Espagne. Il faut signaler également le fait que certaines notations des Carnets $d u$ Front populaire (2006) de Malraux seront reprises, avec quelques changements, dans L'espoir. Les relations de Malraux avec l'Espagne, que Robert S. Thornberry a étudié dans André Malraux et l'Espagne en 1977, sont à la fois étroites et fécondes du point de vue littéraire. Mais elles se sont davantage incarnées dans L'espoir que certains critiques, à sa parution, ont considéré comme un reportage sur la guerre d'Espagne. Mais nous voudrions concentrer notre analyse sur un aspect particulier du tissu romanesque de L'espoir, à savoir le fonctionnement du discours des personnages et du narrateur.

Il s'agira ainsi de montrer d'abord l'importance du dialogue dans ce roman, le dialogue qui permet, parallèlement aux scènes d'action, de réfléchir sur l'action révolutionnaire en cours. Ce dialogue permet ainsi de poser le débat de l'engagement ou de la neutralité de l'artiste, de l'intellectuel et de l'écrivain face à la guerre d'Espagne. Ensuite, il sera question de mettre en lumière la conséquence de cette approche dialogique du roman, c'est-à-dire l'éclatement de la narration. Celle-ci ne se fonde plus sur l'omniscience du narrateur mais plutôt sur une focalisation interne multiple. Le passage du simple reportage journalistique au roman s'effectue donc, dans L'espoir, par le biais des transformations que Malraux fait subir au discours romanesque

\section{L'ART DU DIALOGUE}

Dans L'espoir, le dialogue qui offre le plus de concentration dramatique est probablement celui qui réunit Scali et Alvear et se déroule au chapitre 7 de la section «Etre et Faire». Il met en confrontation deux intellectuels dont l'un (Alvear) a été professeur d'histoire de l'art et l'autre (Scali) a écrit des livres sur l'art. Le sujet du débat est la révolution espagnole et le prochain assaut des fascistes contre la ville de Madrid, d'autant qu'à la différence d'Alvear Scali participe au combat des républicains aux côtés du fils d'Alvear, Jaime Alvear. Malgré leur passion commune pour l'art et leur formation intellectuelle similaire, leur dialogue est celui du combattant et de l'humaniste et concerne essentiellement la question de l'engagement militaire. 
Cette confrontation entre les deux intellectuels est caractéristique de ce renouveau de la discussion sur les grandes pensées de l'histoire et sur les grands idéaux, que les pauses de l'action révolutionnaire ménagent aux personnages malruciens et que Maurice Blanchot ${ }^{2}$ considère comme le signe distinctif des romans de Malraux. Elle est donc l'occasion d'un conflit entre les idées et l'action et se traduit par une double opposition. Il existe d'un côté une divergence idéologique au niveau du discours entre les deux interlocuteurs et de l'autre un décalage entre leurs paroles, leurs gestes et leurs pensées.

Sur le plan de la parole, Alvear clame son indifférence à la révolution espagnole, dénonce la mort qui accompagne la guerre et refuse catégoriquement de quitter Madrid, alors que Scali tente de le convaincre de s'enfuir et lui représente le caractère suicidaire de son refus face à la menace fasciste sur Madrid. Devant la réalité de la révolution, Alvear préfère se réfugier dans les 'nourritures' de l'imaginaire, c'est-àdire la sculpture, la peinture, la littérature, la musique et l'alcool. Cette évasion dans l'art et les paradis artificiels permet certes à Alvear d'oublier les malheurs du monde et le protège de la souffrance mais constitue en même temps une autre forme de fuite plus pernicieuse que l'exil. C'est en effet un signe d'impuissance et une forme de démission. C'est d'ailleurs la raison pour laquelle Scali remet en cause cette sécurité qu'offre le monde de l'art en démontrant à Alvear que la douleur humaine discrédite toute représentation artistique, que l'art ne peut pas ignorer le réel et que l'art pour l'art est un pur phantasme:

Les fascistes savent que votre fils (Jaime Alvear) est combattant...Vous vous rendez compte que vous risquez d'être fusillé? [...]-J'ai acheté la fine. [...]-Vous vous laisseriez tuer par indifférence ? -Pas par indifférence... [...]-Dans les églises du Sud [...], j'ai (Scali) vu en face des tableaux de grandes taches de sang. Les toiles ...perdent leur force... -Il faudrait d'autres toiles, c'est tout, dit Alvear [...]-Bien, dit Scali: c'est mettre haut les œuvres d'art pas les œuvres : l'art. Le plus pur de nous, ce ne sont pas toujours les mêmes œuvres qui permettent d'y accéder, mais ce sont toujours des œuvres. ( André Malraux, L'espoir in Romans, Paris, Gallimard, Bibl. de la Pléiade, 1976, 700-701)

En matière d'idéologie, le dialogue entre Scali et Alvear oppose donc le tenant du progressisme et du militarisme au défenseur du conservatisme intellectuel. De plus les arguments utilisés par les deux débatteurs sont exclusivement individuels et mettent directement en cause la personne et interpellent la conscience de l'interlocuteur. C'est probablement une façon de montrer que les hommes sont non seulement responsables mais aussi qu'ils passent avant leurs idées. Au premier point de divergence qui touche à l'imminence de la menace fasciste sur Madrid, s'ajoute un second point de désaccord qui concerne non plus les deux hommes mais plutôt leurs idéologies. Il s'agit pour Alvear et Scali de définir et de confronter leurs visions de la révolution et de la guerre. Le dialogue prend un tour plus conceptuel, même si les deux interlocuteurs s'engagent entièrement dans la défense de leurs thèses.

D'un côté Alvear recourt à des paraboles de type biblique pour à la fois faire l'éloge de la préservation de la «qualité de l'homme», pointer les illusions qui 
entourent l'engagement de Scali et de son fils Jaime Alvear, fustiger les conséquences désastreuses de la révolution en termes de servitude économique et politique et de cécité de son fils et, en outre, prend ses distances avec le nouvel «âge du fondamental» qui commence avec l'avènement de l'idéologie révolutionnaire. Ce caractère théorique de l'argumentation et ce recours aux paraboles sont la manifestation de ce goût des idées et de ce sens de la formule caractéristiques de Malraux et des intellectuels dans ses romans et une preuve de l'importance des aphorismes dans les dialogues malruciens, ainsi que le souligne Jean-Pierre Blin. ${ }^{3}$ Le point de vue $\mathrm{d}^{\prime}$ Alvear est essentiellement humaniste car il place l'homme, l'éducation, la morale et les valeurs humaines audessus de la guerre, de l'économie, de la politique et des mensonges idéologiques. Et l'exemple de la cécité de Jaime Alvear donne une dimension palpable à son idéalisme et du poids à son message grâce au potentiel d'émotion de cette illustration de sa thèse, même si l'image de son fils provoque de la souffrance chez Alvear, le ramène à sa réalité de père et le met en contact indirect avec la révolution et la tragédie de la guerre.

D'un autre côté, Scali défend la valeur et la légitimité de la révolution. A l'opposé du modèle humaniste d'Alvear, il montre comment la fraternité entre les combattants révolutionnaires secrète des valeurs aussi nobles que celles de l'art. Sur le plan moral, l'espoir révolutionnaire participe à l'élévation de la qualité de l'homme et rivalise à ce titre avec la culture sur le terrain de l'humanisme. Scali démontre ainsi que, dans le domaine de l'intemporel et des valeurs éternelles, qui est celui dans lequel se place Alvear, l'héroïsme a le même statut en termes d'éducation et de transformation de l'homme que la création artistique. Le combattant révolutionnaire parmi ses camarades et au sein de son groupe de combat vaut l'intellectuel au milieu de ses livres:

La révolution joue, entre autres rôles, celui que joua jadis la vie éternelle [...].-L'homme n'engage dans une action qu'une part limitée de lui-même; et plus l'action se prétend totale, plus la part engagée est petite. [...]-Vous avez parlé tout à l'heure de l'espoir : les hommes unis à la fois par l'espoir et par l'action accèdent, comme les hommes unis par l'amour, à des domaines auxquels ils n'accéderaient pas seuls. [...]-[...] L'âge du fondamental recommence, monsieur Scali, dit Alvear [...]. (L'espoir in Romans, pp.704-705)

Autant Alvear illustrait son discours par l'exemple de son fils, autant Scali puise directement à la source de son expérience révolutionnaire. Cette caution personnelle donne du crédit à sa parole et à ses idées sans pour autant céder, comme Alvear, à la recherche de l'émotion. Ainsi s'affrontent deux visions du monde dont l'une défend l'engagement militaire et la nécessité de la révolution et l'autre repose sur le pacifisme et la préservation d'une culture humaniste. Ces deux conceptions idéologiques tranchées sur le plan du discours sont totalement inversées voire nuancées sur le plan des gestes et des pensées de Scali et d'Alvear. En effet les interventions du narrateur mettent en exergue un écart entre la parole $d^{\prime}$ une part et la gestuelle et la psychologie des deux personnages d'autre part. Chez Alvear, ce sont la voix et les gestes qui prédominent. Du point de vue de la voix, il existe un savant dosage de modulation du ton et d'écoute.

Le vieillard s'adonne à une lecture des Sonnets de Quevedo ou à des citations des tragiques grecs en augmentant ou en baissant le volume de sa voix, en accélérant

3 Jean-Pierre Blin, «L'aphorisme dans les romans» in Europe, n727-728,1989, pp.93-103. 
ou en ralentissant le rythme, en modulant l'accent et en recourant au murmure. En ancien professeur, il joue sur toutes les ressources de la voix et toutes les subtilités de la diction afin de toucher et d'émouvoir son interlocuteur Scali. A ce travail sur la voix, il ajoute des phases de pause et d'écoute de la musique de l'Internationale, des bruits de pas et des tirs de mitrailleuses dans la ville de Madrid:

Il (Alvear) tira de sa bibliothèque un autre livre et lut à haute voix trois vers du sonnet de Quevedo: Qué pretende el temor desacordado/De la que a rescatar piadosa viene/Espíritu en miserias anudado? L'index qui suivait les vers faisait reparaître le professeur; assis [...], dans ce fauteuil et dans la poésie, il lisait avec lenteur, avec un sens du rythme d'autant plus saisissant que la voix était sans timbre, aussi vieille que son sourire. (Ibid., p.702)

Grâce à ce mélange de parole et de silence, Alvear donne de la variété et de la richesse à son discours pour le faire échapper à la monotonie d'une leçon et retenir ainsi l'attention de Scali. En outre, cette stratégie montre qu'Alvear est, en dépit de son point de vue humaniste, sensible et réceptif à la révolution et à ses éclairs de beauté, c'est-à-dire à sa musique. Quant aux gestes d'Alvear, ils se répartissent en deux catégories. La première catégorie concerne l'acuité des regards posés sur Scali. Ces regards insistants voire théâtraux du vieillard sont destinés à fixer l'attention de l'interlocuteur et à percer à jour son mystère en pénétrant le fond de son âme. Ils témoignent aussi d'un sens aigu de l'observation et de la physionomie héritée de son métier d'expert en sculpture, même s'ils peuvent apparaître par ailleurs comme une déformation professionnelle.

On dirait que Scali devient un objet d'étude pour Alvear au même titre que ses œuvres d'art et ses livres car il s'aide d'un lorgnon et lève son index pour à la fois scruter Scali et désigner les objets de sa bibliothèque. Le lorgnon et la levée de l'index accréditent d'ailleurs son rôle d'homme d'étude car ses accessoires sont des signes distinctifs de presque tous les intellectuels de Malraux. La seconde catégorie a trait à la marche et aux déplacements d'Alvear à l'intérieur de sa chambre. En effet non seulement il se rend à sa bibliothèque pour chercher les CEuvres complètes de Cervantès et se verse des verres de fine mais aussi il ouvre sa fenêtre pour observer Madrid et recueillir les bruits de la ville:

Alvear se leva à demi, ne quittant pas des mains les bras du fauteuil, et regarda Scali un peu
théâtralement, comme pour souligner ce qu'il disait [...] Scali comprit enfin ce qui le troublait
depuis le début de l'entretien : toute l'intensité du visage du vieillard était dans ses yeux [...]
Il (Alvear) se leva, chercha l'édition des CEuvres complètes de Cervantès, ne la trouva pas. [...] Il
posa Quevedo sur un rayon. Scali n'avait pas envie de s'en aller. (Ibid., pp.701-702)

Cette activité du vieillard corrige l'image de passivité que l'importance de la voix et des regards pouvait véhiculer de lui en tant qu'intellectuel et démontre au contraire sa disponibilité, sa curiosité à l'égard du monde et son engagement dans la cause humaniste. Ce goût du mouvement est aussi une remise en cause de sa vieillesse et peut-être une manière de souligner l'inutilité de l'aide à l'exil de Scali. Cette impression de solidité, de validité, de maîtrise de la voix et du corps modifie l'image anachronique et rêveuse d'humaniste et d'artiste que laissait supposer le discours d'Alvear et lui confère une autre stature. Alvear apparaît ainsi comme le prototype de 
ces intellectuels «chaman», dont Monique Gosselin étudie la présence dans les romans de Malraux, en ce sens qu'il ne communique pas un savoir purement rationnel à Scali mais plutôt un secret de vie dont il se porte physiquement garant. Tout se passe comme si Alvear et Scali avaient échangé leurs positions en ce sens qu'Alvear apparaît comme un intellectuel combattant pour ses idées et qu'au contraire Scali paraît démissionner de l'action révolutionnaire et retomber dans les ornières de l'intellectuel passif.

Cette situation est d'ailleurs confirmée par le fait que le narrateur ne transmet de Scali que principalement ses pensées, à l'exception d'une courte mention relative à sa gestuelle. D’une manière générale, les pensées de Scali ont trait à ses introspections et traduisent ses démons intérieurs. Soit elles font état d'un besoin de compréhension et de communion avec Alvear sur la base de leur univers intellectuel commun, soit elles révèlent les angoisses de Scali qui perçoit dans les regards d'Alvear la ressemblance du père avec le fils Jaime et l'image du destin en raison de la cécité de ce dernier. Scali prend en outre conscience de l' «élément physiologique de la guerre» à partir du discours d'Alvear sur la douleur, discours dont il ignore s'il s'agit d'un autoportrait du vieillard ou d'une expression objective de sa pensée:

Alvear touchait en Scali un ordre d'expériences qu'il ignorait, et qui devenait tragique chez
le petit Italien frisé. Pour Scali, ce qui menaçait la révolution n'était pas le futur, mais bien
le présent : depuis le jour où Karlitch l'avait étonné, il voyait l'élément physiologique de la
guerre se développer chez beaucoup de ses meilleurs camarades, et il en était atterré. [...] Scali
se demandait s'il était en face de la pensée d'Alvear ou de sa douleur. (L'espoir in Romans,
pp.704-705)

Les pensées de Scali sont donc totalement coupées de la révolution et ne transmettent plus que les peurs de son inconscient. Et elles dégagent une impression générale de fragilité et de terreur qui contraste avec l'engagement et l'éloge de la révolution effectué par Scali dans son discours. Autant les gestes et la voix d'Alvear raffermissent sa parole, autant les pensées de Scali creusent un fossé avec son action. Cette emprise de l'inconscient sur Scali empêche d'ailleurs toute vraie communication avec Alvear et tend à le séparer de la communauté révolutionnaire. De plus, le seul geste important qu'il effectue consiste à remplacer Alvear sur son divan. Ce qui est un geste d'impuissance et de passivité caractéristique de beaucoup d'intellectuels malruciens.

Il existe d'ailleurs un lien et une continuité entre l'installation dans le divan et les pensées de Scali car le retour de l'inconscient et l'abandon à l'introspection coïncident presque parfaitement avec l'introduction du divan dans le dialogue. On pourrait presque dire que Scali n'est plus combattant mais est devenu un cas psychanalytique car chez Malraux l'inconscient est souvent associé à la folie. Cette inversion des rapports et des rôles entre Alvear et Scali est symptomatique de l'influence du discours encadrant du narrateur sur le dialogue des personnages car les gestes, les jeux avec la voix et les pensées nuancent ou contredisent les paroles des débatteurs. Cette situation

4 Monique Gosselin, «Le rêve malrucien de l'intellectuel 'chaman'» in L'intellectuel et ses miroirs romanesques (1920-1960), Presses Universitaires de Lille, 1993, pp.97-117. Cet intellectuel «chaman» symbolisé par Alvear s'oppose, dans les romans de Malraux, à l'intellectuel engagé représenté par Scali. 
nous amène évidemment à nous intéresser au fonctionnement autonome du récit et plus globalement du roman malrucien en rapport avec la théâtralité.

Chez Malraux, le dialogue est un élément majeur de l'architecture romanesque. En effet, les scènes de dialogue succèdent souvent aux scènes d'action et servent à réfléchir sur la stratégie militaire ou sur les idées au nom desquelles les personnages combattent. Il existe donc au cœur des romans malruciens cette volonté de maintenir le lien de la communication, y compris entre des adversaires politiques. Cette construction du dialogue est présente également dans le débat idéologique entre les personnages. Dans ce débat, ce ne sont pas les idées qui nous intéressent comme dans la discussion philosophique mais plutôt leur mise en situation conflictuelle. Le premier élément de cette dramatisation des idées a trait à leur incarnation par les débatteurs. Tandis que la présence des intellectuels comme Alvear risquait de cantonner le débat à la discussion des grands idéaux comme la justice, la paix ou l'art, les héros révolutionnaires tels que Scali ramènent l'échange sur le terrain de l'action en invoquant leurs expériences personnelles de combattant et en faisant intervenir l'exemple d'un troisième personnage, à savoir le fils aveugle d'Alvear (Jaime).

Ce qui au-delà de la différence idéologique situe le débat au niveau non pas du passé ou de l'éternité mais à celui du présent et des individus vivants. Les idées n'ont ainsi d'existence que prises en charge par des individualités marquées, même si les débatteurs sont en désaccord puisque le roman ne tranche pas clairement entre les positions idéologiques de Scali d'un côté et d'Alvear de l'autre. Le second élément de dramatisation du débat, qui est une conséquence du premier, tient au fait que l'incarnation des idées ne se fait pas seulement par la parole mais aussi par les gestes et les pensées qui forment comme au théâtre un appareil didascalique entourant la prise de parole des personnages. En effet, les intellectuels font davantage appel aux modulations de la voix, à la diction, aux regards, à l'écoute, aux mouvements des mains et à la pensée que les combattants qui ne recourent que accessoirement à la pensée et principalement aux gestes de déférence.

Ainsi se fait jour une totale dissymétrie dans le débat entre la variété de l'expression orale et corporelle des intellectuels et la pauvreté des ressources discursives des hommes d'action. Cette dissymétrie traduit certes l'inexpérience des seconds dans le maniement du discours mais elle met surtout en lumière le désaccord entre les personnages. Elle peut aussi signifier que les intellectuels ont besoin de jouer un rôle pour donner du poids et de la chair à leur parole qui est essentiellement coupée du monde, alors que les révolutionnaires peuvent faire preuve d'une économie des moyens puis que leur expérience militaire parle pour eux. Mais l'accompagnement de la parole par les jeux sur la voix, les mouvements, la gestualité et l'exposition des pensées démontrent que le débat idéologique dans les romans de Malraux est aussi polysémique que celui du théâtre car la communication s'établit grâce à la conjugaison de la parole et du corps. 


\section{NARRATION ET FOCALISATION INTERNE MULTIPLE}

Contrairement aux Conquérants et à La condition humaine, L'espoir donne à voir une narration dans laquelle la focalisation interne est tellement étendue que le récit présente une fragmentation extrême. Les personnages-réflecteurs se multiplient et ne défendent plus seulement des points de vue individuels mais sont souvent les porte-parole des tendances politiques ou idéologiques de L'espoir. Cet émiettement du récit conduit à la disparition ou à tout le moins au remplacement du narrateur par le personnage de Garcia. Ce changement ne signifie pas un retour à la narration omnisciente des Conquérants mais obéit plutôt au besoin d'intelligibilité du texte. Car Garcia préserve la dramatisation du récit par le partage de la vision limitée des personnages et par son implication dans l'histoire de la révolution espagnole et en même temps donne de la cohérence et du sens à la narration. Ce personnage assume à lui seul donc les fonctions traditionnelles du narrateur, c'est- à dire informer, donner du sens et opérer une synthèse. ${ }^{5}$

Cette position centrale de Garcia est particulièrement remarquable dans deux séquences de la première partie (chapitre 3 de la troisième sous-section de «L'illusion lyrique» et chapitre 4 de la deuxième sous-section $\mathrm{d}^{\prime}$ »Exercice de l'Apocalypse») de L'espoir qui mettent en scène d'un côté le trio Garcia, Magnin et Vargas, et de l'autre Garcia, le groupe Shade-le Négus-Hernandez et le groupe Manuel-Pradas-Golovkine. Dans les deux cas, Garcia joue le rôle d'un tiers personnage qui à la fois participe à la confrontation des visions et synthétise les points de vue, à l'intérieur d'un schéma triangulaire au sein duquel il sert de conscience à l'expérience révolutionnaire conflictuelle entre le peuple représenté par Magnin, Shade, le Négus et Hernandez et l'armée incarnée par Vargas, Pradas et Golovkine.

Cette position centrale de Garcia se traduit dans les deux séquences de L'espoir par une distanciation et une mise en valeur de la voix de Garcia par rapport à celles des autres personnages réflecteurs. Ainsi dans la première séquence, l'on passe, dans la présentation des personnages et de l'action révolutionnaire, $d^{\prime}$ un changement constant de voix et de vision à une focalisation sur le personnage de Garcia. En effet, les trois protagonistes de la scène du ministère de la guerre sont vus à travers les yeux les uns des autres. Vargas est vu par Magnin selon un angle ironique car sa ressemblance physique avec Don Quichotte et son uniforme contrastent avec l'expérience militaire commune des deux personnages dans les lignes aériennes espagnoles et le sabotage des rails du Séville-Cordoue.

De même, c'est Magnin qui voit Garcia et relève le décalage entre son physique et son statut d'intellectuel, entre son discours et sa fonction dans la révolution espagnole, en ce sens que son type anglais, son absence de bronzage et de binocles et sa volubilité remettent en question son prestige d'ethnologue et de chef des renseignements militaires et son devoir de réserve. Quant à Magnin, il n'est vu et présenté que par

5 Philippe Carrard, Malraux ou le récit hybride. Essai sur les techniques narratives dans L'espoir, Paris, Lettres Modernes-Minard, 1976. (Chapitre 3 'L'Action': 2-L'instance narrative et l'action). 
le biais de Garcia qui soupçonne, chez lui, le mélange d'ébéniste du faubourg SaintAntoine et d'intellectuel mais n'en perçoit que la fonction de technicien de guerre:

Tout de Garcia surprenait Magnin : que cet Espagnol eût [...] ce visage de grand propriétaire terrien anglais ou normand [...]; que cet intellectuel eût l'air rigoleur et si cordial [...]. (L'espoir in Romans, p.525) 'Garcia observait Magnin avec soin. [...] Et tout de Magnin l'intéressait : [...] son aspect de contremaître supérieur [...]. Il y avait en Magnin [...] quelque chose de l'ébéniste traditionnel du faubourg Saint-Antoine [...], la marque complexe de l'intellectuel. (Ibid., p.526)

Ces portraits croisés, outre le fait qu'ils présentent Garcia et Vargas en fonction de leur personnalité (bouffonnerie, verbiage) et Magnin en fonction de sa fonction, renforcent le point de vue de Garcia car il détient le monopole à la fois de la vision sur Magnin et des renseignements militaires et tient sous sa dépendance le directeur des opérations militaires (Vargas) à qui son aspect caricatural enlève toute crédibilité. À la vision éclatée de Magnin écartelé entre Garcia et Vargas, s'oppose la vision unifiée de Garcia qui annexe celle de Vargas. La présentation des personnages par la focalisation interne variable met déjà en évidence les deux points de vue qui s'affrontent dans le discours des trois personnages: le point de vue militaire et le point de vue de l'illusion populaire, le point de vue de Vargas n'étant là que pour affaiblir celui de Magnin.

À cette focalisation changeante sur les visions respectives de Magnin, de Vargas et de Garcia, succède une focalisation unique sur Garcia. Ainsi le passage du portrait des personnages à l'évocation de la révolution espagnole concentre le récit autour de la lecture des rapports des renseignements militaires en provenance de Badajoz par Garcia. Non seulement, la narration met l'accent sur la lecture des trois rapports qui font état de l'attaque et de la prise de Badajoz par les forces fascistes, mais aussi elle commente les pauses de Garcia. Les deux premiers rapports sont chronologiques (5h, $7 \mathrm{~h}, 9 \mathrm{~h}, 11 \mathrm{~h}, 12 \mathrm{~h}, 16 \mathrm{~h}, 16 \mathrm{~h} 10)$ et elliptiques et mentionnent successivement les prises du fort de San Cristobal, la destruction des casernes et des remparts de l'hôpital de Badajoz, l'affrontement entre l'artillerie et les tanks de Franco et les mitrailleuses des milices républicaines, l'attaque de la cathédrale, l'entrée des Maures dans la ville et le début des combats de rues.

Le troisième rapport, lui, est dénué de chronologie, présente une origine floue et évoque l'arrestation, l'inculpation et l'exécution des miliciens républicains et la libération des prisonniers politiques fascistes. Cette lecture des rapports de Badajoz est accompagnée des commentaires critiques de Garcia sur la capacité des milices et des mitrailleuses républicaines à résister à l'infanterie maure, sur l'origine et l'heure d'émission du troisième rapport. À ces commentaires émanant de Garcia lui-même s'ajoutent d'autres qui ponctuent les pauses et soulignent les effets de sa lecture. En effet, l'hymne républicain, les chants du peuple, les «salud» et les notes de piano d'un côté et le hochement de tête ou le haussement des sourcils de Vargas de l'autre apportent des éléments de contradiction à la lecture et au commentaire de Garcia:

Par la fenêtre ouverte, l'hymne républicain diffusé par vingt radios entrait [...]-Je continue, dit Garcia reprenant son rapport : c'est Badajoz ce matin [...]. 5 heures. Les Maures viennent d'entrer dans le fort de San-Cristobal [...]. 7 heures. L'artillerie ennemie [...] bombarde la ville sans interruption. [...] Il posa la feuille dactylographiée, en prit une autre. [...] 12 heures. Les tanks sont à la cathédrale. [...] 16 heures. L'ennemi entre. 16h.10. On se bat maison par maison. [...]-Voici le dernier rapport, dit 
Garcia. [...] Les miliciens et suspects arrêtés sont passés par les armes. [...] On a fusillé tout l'après-midi. Les fusillades continuent. (Ibid., pp.527-528)

Ce qui prouve que la lecture de Garcia s'enracine dans l'actualité de la révolution espagnole et résume le sens de l'affrontement entre les différentes tendances: les Maures et les Républicains, le peuple et l'armée (Vargas). Garcia est donc le porteur d'information car il éclaire la bataille de Badajoz par la réception et la lecture des rapports. Son discours est également doublement commenté par lui-même et par des notations extérieures sur les réactions à sa lecture. Garcia se constitue ainsi un auditoire par la centralisation et la communication des renseignements militaires. Par conséquent, les opérations militaires (Vargas) et l'aviation républicaine (Magnin) dépendent de ses informations.

Par la lecture, Garcia participe à la focalisation interne grâce à la nouvelle vision des rapports qui présentent des lacunes au même titre que la vision des personnages (Magnin, Vargas), mais en même temps prend ses distances avec le point de vue des renseignements grâce aux critiques, aux commentaires directs ou indirects et à l'interprétation des événements. C'est par la connaissance davantage que par la vision que Garcia, contrairement aux autres personnages, accède au statut de narrateur car $c^{\prime}$ est lui qui donne du sens à la révolution, analyse et synthétise les points de vue contradictoires qui s'affrontent. Et ce n'est pas un hasard si Magnin et Vargas l'écoutent comme des disciples écoutent leur maître.

L'affirmation du rôle de personnage narrateur de Garcia est beaucoup plus visible dans la seconde séquence qui se déroule au musée de Santa-Cruz. Par rapport à la première séquence, celle-ci repose sur une focalisation interne qui partage la vision et la voix entre une multitude de personnages et rétrécit d'autant le champ de leur perception. Elle constitue ainsi un «cadre participatif polylogal» ${ }^{6}$ caractérisé par une mobilité des places interlocutives, une variation des instances narratives et un mélange entre dialogue et narration. De plus, les réflecteurs ne sont plus, en raison de la fragmentation des points de vue, des personnages individualisés mais des groupes idéologiques et politiques, à l'exception notable de Garcia. Ainsi, une phase de focalisation tournante entre le groupe Hernandez-Shade-le Négus- Mercery et le groupe Pradas-Manuel-Golovkine cède la place à une phase où le récit se resserre et est pris en charge par Garcia.

Dans la première phase, les changements de point de vue sont préparés par le gros plan sur le désordre des miliciens républicains. C'est la terreur de Pancho Villa, le jeu à la garde d'Hernandez, le sommeil des miliciens, l'absence de protection d'Hernandez face à Shade et Golovkine et l'abondance du soleil qui sont l'objet de la description et donnent au musée de Santa-Cruz l'aspect d'un lieu de villégiatures et aux miliciens l'allure de vacanciers. Ce qui donne aussi une impression d'amateurisme et d'absence de commandement dans les rangs des Républicains. Cette dispersion des miliciens se traduit par une dislocation de la vision et de la voix narratives. Ainsi

6 Cf. Francesca Cabasino, «Le mythe révolutionnaire entre dialogue et monologue dans L'espoir d'André Malraux» in Mots, n54, Mars 1998, pp.58-59. 
les points de vue de Shade et du Négus qui sont les porteurs de vision et de voix du premier groupe d'un côté et les points de vue de Pradas et de Golovkine qui portent la voix du second groupe de l'autre s'affrontent autour d'Hernandez, au sujet de sa transmission de la lettre du colonel fasciste Moscardo à sa femme durant l'Armistice de l'Alcazar de Tolède.

Dans ces deux couples, ce sont le Négus et Pradas qui sont les réflecteurs principaux, les deux journalistes Shade et Golovkine étant le contrepoint ironique et Hernandez le témoin. Le Négus défend les valeurs de générosité, de courage, de sacrifice, de solidarité avec le prolétariat et de primauté de l'homme sur les appareils (Etat, Armée, Parti), fait état de sa fascination pour la statue du soldat inconnu et dénonce la caricature du syndicalisme espagnol, de la grève générale et de l'anarchisme par le communisme. C'est une vision idéale, humaniste, morale et chevaleresque de la révolution. Elle est d'ailleurs mise en lumière par les notations sur la gestuelle du Négus et les références chrétiennes de son discours, notamment la levée de la «main du Christ enseignant», la mise à l'index d'une crucifixion à la Bonnat fusillée par les fascistes et les gestes d'encouragements des statues de saints. Le point de vue du Négus est donc placé sous le signe de la religion et souligne son fanatisme et son idolâtrie de l'anarchisme. Quant à Pradas, il est le chantre de la discipline, de la transformation de la société, de la primauté de l'action sur la morale, de l'efficacité sur la justice et de la supériorité du groupe sur les individus et dénonce la prédication des anarchistes. Son point de vue se place sous le patronage du Parti communiste et est conforme aux indications sur l'écoute attentive et la traduction méthodique du discours du Négus grâce notamment à la main sur le crâne:

Le Négus montrait de son index court une crucifixion à la Bonnat [...], fusillée depuis des jours par ceux d'en face. [...]-Si nous sommes écrasés ici et à Madrid, les hommes auront un jour vécu avec leur cœur. [...] Pradas passa sa main jusqu'au sommet de son crâne en poire, attentif. [...] Le Négus leva la main droite avec le geste du christ enseignant : -Celui qui a peur de mourir n'a pas la conscience tranquille. [...]-En dernière analyse, vous êtes des chrétiens, dit Pradas professoral. (L'espoir in Romans, pp.602-603)

Face à ces deux points de vue contradictoires et sectaires, se tiennent les points de vue d'Hernandez, de Shade et de Golovkine. Alors que le discours et les commentaires sur Shade et Golovkine portent respectivement sur l'indifférence à l'opposition entre l'anarchisme et le communisme, sur la gourmandise, sur la dérision de la religion, sur l'incompréhension du discours du Négus et sur la curiosité pour la transmission de la lettre de Moscardo par Hernandez, celui-ci ne fait l'objet que de brèves notations sur ses mouvements de tête ou de mains ou sur la démesure de son corps.

Ces trois points de vue introduisent, dans le conflit idéologique Pradas-Négus, une distance ironique par le silence, par des considérations sur le végétarisme du Négus, sur des souvenirs familiaux dans l'Iowa-City, sur la fourchette à l'effigie des armes de l'archevêché et le chapeau de cardinal de Shade. Ils témoignent d'un parti pris d'indifférence aux idéologies et de retour au bon sens paysan et remettent en cause l'importance des points de vue du Négus et de Pradas. Et ce n'est pas un hasard 
si Shade se signale par ses interventions intempestives contre le sectarisme de Pradas et la prédication du Négus:

\begin{abstract}
Moi, dit Shade, suave, les deux mains accrochées aux pompons du chapeau, je m'en fous. Ce que vous faites les uns et les autres est plus simple et mieux que ce que vous dites. Vous avez tous de trop grosses têtes. Dans ton pays, d'ailleurs, Golovkine, tout le monde commence à avoir une grosse tête. [...] Je trouve le Négus un peu connaud, mais il me plaît. L'atmosphère se détendait. Hernandez regarda de nouveau sa montre, puis sourit. Ses dents étaient longues, comme ses mains et son visage. (Ibid., p.604)
\end{abstract}

Cette coexistence de plusieurs points de vue égalise et rétrécit le champ de toutes les visions et menace même la compréhension du récit à force de ramification. C'est également un signe du fait que « les héros de L'espoir, en dépit du titre, sont beaucoup moins sûrs, non pas des valeurs qu'ils défendent, mais de l'avenir de ces valeurs». $C^{\prime}$ est dans ce contexte où toutes les visions se valent et s'opposent qu'intervient Garcia qui redonne de l'unité et de la signification à cette narration confuse en raison de son éclatement. Et le passage des changements incessant de focalisation entre les différents groupes du musée à la cristallisation de la focalisation est mis en scène et thématisé.

En effet, l'entrée de Garcia attire d'abord les regards de tous les personnages et provoque une réorganisation de l'espace avec les mouvements de table. Ensuite, elle met fin au discours du Négus, dans la mesure où l'abandon de son revolver par celuici et le dépôt de la pipe de Garcia sur la table sont concomitants. La simultanéité de ces deux gestes est le signe d'un passage de témoin, en ce qui concerne la voix narrative, du Négus à Garcia. De plus, c'est Garcia qui prend en charge la traduction en russe du discours du Négus pour Golovkine et en espagnol des questions de Golovkine adressées à Manuel:

On faisait place à Garcia qui venait d'arriver, pipe au coin du sourire. [...] L'œil d'écureuil aux aguets de Garcia s'alluma. [...] Une belle occasion de se taire perdue, pensa Garcia. [...] Golovkine ne comprenait plus, mais des mots saisis çà et là l'inquiétaient. Garcia lui dit quelques mots en russe. [...] Le Négus tira son revolver et le posa sur la table. Garcia posa sa pipe de la même façon. (L'espoir in Romans, pp.602-603)

De ce fait, Garcia récupère le privilège de la traduction que détenait Pradas. Ainsi, Garcia monopolise les attributs de la voix et de la vision narrative et rétablit la communication entre les différents points de vue grâce à la traduction. L'information comme la focalisation sont désormais entre les mains de Garcia dont l'apparition homogénéise et réduit les points de vue à ceux de Manuel, Pradas, Golovkine, c'està-dire tous les personnages dépendant indirectement du renseignement militaire et sensibles au rôle de l'armée. Cette disparition du point de vue anarchiste ou libéral (le Négus, Hernandez, Mercery, Shade) préfigure peut-être le dépassement de 'l'illusion lyrique' par l'érection d'une armée et la réduction du roman au point de vue de L'espoir. Garcia apporte une troisième dimension au récit en synthétisant les points de vue, en conférant de la cohérence et de la simplicité à la focalisation interne et en donnant du sens aux différentes visions des personnages réflecteurs.

7 Jean Carduner, «L'espoir ou la fin de l'imaginaire de roman» in Revue d'histoire littéraire de la France, $\mathrm{n}^{\circ} 2$, mars-avril 1981, pp.236-247. 
Et même si de la première à la deuxième séquence, l'auditoire change de nature (Magnin-Vargas d'un côté, Manuel-Pradas-Golovkine de l'autre), les fonctions d'information et d'interprétation de Garcia restent les mêmes, car le commentaire des rapports et la traduction, les renseignements de Badajoz et du Négus se valent. Garcia participe donc à la confrontation des points de vue des personnages par l'écoute du discours du Négus et s'en démarque par la traduction du message pour Golovkine. Il satisfait ainsi pleinement au critère de participation-distanciation propre au narrateurpersonnage. Le sens de la transmission de la lettre de Moscardó par Hernández et du conflit idéologique entre les anarchistes et les communistes sera éclairci par Garcia car c'est lui qui a en charge de donner voix au silence et aux gestes d'Hernández et signification au fanatisme du Négus et au sectarisme de Pradas, à travers notamment son discours où percent des interventions de l'auteur.

\section{NARRATION ET VOIX DE L'AUTEUR}

A l'instar de sa lecture et de sa traduction, le discours de Garcia participe à son travail d'interprétation et à sa position de personnage-narrateur. Il s'agit, pour lui, de prendre du recul par rapport à l'action et aux renseignements militaires, d'évaluer les idéologies, les hommes, les valeurs et les points de vue contradictoires et de donner une vue d'ensemble et une vision intellectuelle des événements. C'est la traduction en conscience de l'expérience révolutionnaire. Ce qui rappelle le projet d'écrivain de Malraux qui ne manque pas d'intervenir dans le récit. En effet, la voix de l'auteur interrompt périodiquement le discours de Garcia soit pour le compléter, soit pour le nuancer, soit pour l'élargir. Par des intrusions ponctuelles sous forme de références culturelles ou historiques, l'auteur se place dans la position du narrateur pour soit prolonger la vision subjective du personnage réflecteur soit pour pallier son incompétence narrative. ${ }^{8}$ Mais l'essentiel de la voix et de la vision est porté par Garcia qui confronte son point de vue à ceux des autres personnages.

Ainsi dans la séquence de «l'illusion lyrique», face à Magnin qui défend la communion du peuple avec les miliciens républicains et la fraternité populaire, refuse le manichéisme politique entre l'Apocalypse et la Révolution et la primauté de la discipline sur les rêves du peuple et brandit l'exemple de la défense de la Sierra par le peuple espagnol, et à Vargas qui, outre le fait qu'il complète les rapports de Garcia par des renseignements relatifs à la formation d'une base aérienne fasciste à Badajoz et prône la constitution d'une armée, ironise sur l'impuissance du peuple, Garcia éclaire les enjeux politiques et symboliques de la révolution espagnole. D'une part, il explique comment l'aviation et l'armée moderne de Franco mettent fin au mythe du peuple victorieux, que véhiculent les révolutions française et russe et qui se traduit en Espagne par les barricades, et commandent le passage à la Révolution grâce aux moyens techniques et à la formation d'une armée avec des cadres.

8 Sur ces interventions de l'auteur pour compléter la vision de Garcia, voir : Philippe Carrard, op.cit, 1976. 
A cette opposition entre les mythes du Peuple et de la Révolution, mise en lumière par Garcia, s'ajoute une lecture géopolitique de la guerre civile espagnole. Garcia montre que l'enjeu de la guerre n'est plus désormais dans les idéologies et les mythes mais plutôt dans la technique et le soutien militaire international. Il démontre que le coup d'Etat de Franco est un non-événement en raison de l'alliance de Mussolini avec les Maures et l'Islam et du projet d'attaque de Madrid par l'armée de Franco soutenue par les colonnes italiennes et les avions allemands:

Mon cher monsieur Magnin, nous sommes soutenus et empoisonnés à la fois par deux ou trois mythes assez dangereux. [...]-Et surtout Magnin, dit Vargas [...]. Jamais des milices ne battront une armée moderne. [...]-Les hommes ne se font pas tuer pour la technique et pour la discipline, dit Magnin. -[...] Quant à ce que nous entendons par la fenêtre, monsieur Magnin, c'est l'Apocalypse de la fraternité. [...] c'est une des choses les plus émouvantes qu'il y ait sur la terre [...]. Mais elle doit se transformer, sous peine de mort. (L'espoir in Romans, p.604)

Les éléments techniques et géostratégiques brisent le manichéisme entre le peuple et la révolution en imposant un nouveau défi et une guerre moderne à l'Espagne. Ce discours de Garcia est d'ailleurs mis en valeur par le recours aux italiques qui isolent certaines expressions terminales de son argumentation. Ces passages en italique mettent l'accent sur la modernité de la guerre contre l'armée de Franco, sa différence avec la guérilla de la Sierra et sur la nécessité d'une transformation de l'A pocalypse par le recours à la technique. Ils jettent une lumière sur les conclusions de Garcia en leur donnant l'allure de vérités générales et rappellent en même temps le style des Oraisons funèbres et l'éloquence de Malraux. Ce sont aussi des tics de langage qui confirme la dimension intellectuelle du discours de Garcia en se focalisant non pas sur l'analyse des événements révolutionnaires mais sur l'interprétation qu'en donne Garcia.

Dans la séquence $\mathrm{d}^{\prime}$ «exercice de l'Apocalypse», alors que Manuel, Pradas et Golovkine s'interrogent, réprouvent la transmission de la lettre de Moscardo par Hernández et expliquent la révolution espagnole par l'humiliation, la pauvreté des masses, la luttes des classes et l'économie, Garcia procède à une double analyse des points de vue du groupe du Négus et du camp de Pradas. D'un côté, il se livre à une analyse psychologique pour mettre à jour à la fois les valeurs de libéralisme, d'éthique, de dignité et de sacrifice qui président au fanatisme du Négus, au geste d'Hernández et à l'Armistice d'Alcazar, et le sens de la comédie et le dilettantisme qui s'attachent aux débuts de toute révolution. Un mélange d'humanisme et d'amateurisme définit donc et explique le point de vue du Négus et les actes d'Hernández.

Mais à cette démonstration psychologique, destinée peut être davantage à rétablir l'équilibre entre les points de vue anarchiste et communiste en soutenant celui des absents (le Négus, Hernández) et l'impartialité du récit, Garcia ajoute une réflexion intellectuelle qui transcende le clivage politique et idéologique entre l'anarchisme et le communisme. Ce manichéisme est, en effet, dépassé par l'introduction et la mise en lumière d'un troisième élément perturbateur: le phénomène des masses. Ce dernier s'oppose aux partis anarchiste et communiste et invalide les explications économiques et psychologiques de Pradas, Manuel, Golovkine et Garcia: 
Garcia commençait à repérer les mots-types des partis : pour les communistes, c'était 'concrètement'. [...] Evidemment, reprit-il, le Négus n'est pas Hernandez ; mais entre libéral et libertaire il n'y a qu'une différence de terminologie et de tempérament. [...]-Les anarchistes, dit Manuel, c'est un mot qui sert surtout à embrouiller. [...]-[...] Il est tout de même temps de se rendre compte que les masses sont une chose et que les partis en sont une autre. [...] Rien n'est plus difficile que de faire penser les gens sur ce qu'ils vont faire. (L'espoir in Romans, pp.608-610)

Comme la politique étrangère, la masse est un élément politique que ni les catégories intellectuelles de Garcia ni les idéologies traditionnelles ne permettent de comprendre et de penser. Mais elle traduit exactement, sur le plan de la révolution espagnole, la position de tiers personnage et d'extériorité de Garcia dans le récit. Sa position distante de narrateur est ainsi thématisée dans son discours. Mais ces éléments nouveaux, qui échappent à la compréhension de Garcia, montrent en même temps les limites de son statut de narrateur et d'intellectuel. En fait, non seulement il est réduit aux hypothèses en ce qui concerne l'avenir de l'alliance entre Mussolini et l'Islam et omet l'analyse politique des anarchistes mais aussi les pièges du discours le coupent de la réalité révolutionnaire et des renseignements militaires et l'empêchent de fournir à l'état-major de Madrid des informations sur les négociations de l'Alcazar entre les fascistes et le prêtre républicain.

Ces lacunes de la vision de Garcia sont comblées par les interventions directes de l'auteur dans son discours. Ces intrusions concernent d'une part le regard que Garcia porte sur les autres personnages et de l'autre sur la psychologie de Garcia luimême. Dans tous les cas, elles se traduisent par des parenthèses à l'intérieur du point de vue et de la voix de Garcia ou par des commentaires extérieurs qui rompent le fil du récit. Ainsi, dans la séquence de «l'illusion lyrique», l'auteur fournit une information sur la formation d'ingénieur à Centrale de Magnin pour confirmer l'impression de «contremaître supérieur» que Garcia a du chef de l'aviation républicaine. Dans la séquence d'«exercice de l'Apocalypse», il élargit les analyses de Garcia sur le rôle d'officier d'Hernandez à tous les officiers de carrière d'Espagne, sur la similitude des conditions économiques des anarchistes, des socialistes et des communistes et la violence de l'anarchie espagnole à tous les amis des anarchistes et au pittoresque inhérent à l'anarchisme:

J'appelle ainsi (la discipline) l'ensemble des moyens qui donnent à des collectivités combattantes la plus grande efficacité. (Garcia avait le goût des définitions.) ; -[...] Les limites de l'anarchie espagnole (le pittoresque dépassé) étaient celles du syndicalisme même, et les plus intelligents des anars ne se réclamaient pas de la théosophie, mais de Sorel. (Ibid., p.530)

Ces interventions de l'auteur apportent des informations supplémentaires au lecteur concernant les personnages et donnent de la révolution une vision plus concrète et populaire en mettant l'accent sur les éléments humains (les officiers, le peuple des sympathisants anarchistes, le sens du comique et du jeu des anarchistes) qui échappent à la vision de Garcia.

L'auteur interrompt donc Garcia pour donner une vision plus complète de la révolution espagnole que celle de ce dernier car les renseignements fournis par l'auteur mettent en exergue les limites du chef des renseignements et les lacunes dues à son statut de narrateur- personnage et à la focalisation interne, même si ces intrusions 
d'auteur se font à travers des incises dans le discours de Garcia et témoignent d'une connivence entre l'auteur et son narrateur.

Mais les liens entre l'auteur et Garcia se distendent, lorsque l'auteur donne des indications sur le personnage de Garcia et donc ne participe pas à son discours et à sa vision mais le voit presque entièrement de l'extérieur. Ainsi, l'auteur fait d'abord deux remarques sur la psychologie de Garcia en soulignant son goût des définitions et sa capacité de conviction. Mais on sent poindre, dans les interventions de l'auteur, une dose d'ironie car il ne s'agit pas de magnifier le statut d'intellectuel et la compétence de Garcia mais plutôt de mettre en valeur la distance entre la passion de l'abstraction de Garcia et l'incompréhension des auditeurs et entre l'absurdité de son discours et la crédulité de son auditoire:

Garcia paraissait d'autant plus intelligent à une partie de ses auditeurs qu'ils devinaient ce qu'il disait plutôt qu'il ne le comprenait. [...] Maintenant Garcia se taisait et réfléchissait. [...] Toute cette conversation (avec Manuel et Pradas) se développait comme si les anarchistes eussent été une race particulière [...], comme si Garcia eût dû les étudier, non en tant que politique, mais en tant qu'ethnologue. [...] Etrange le goût des hommes de discuter d'autre chose que des conditions de leur action, au moment même où la vie est suspendue à leur action. (Ibid., p.610)

Ce qui sert à relativiser et à remettre en question les vérités générales et l'interprétation intellectuelle de Garcia et replace son point de vue parmi les visions limitées des autres personnages réflecteurs. A ces indications psychologiques, succèdent des commentaires de l'auteur sur la pipe de Garcia. Cet accessoire permet à l'auteur de marquer la prise de parole et la fin du discours de Garcia, l'interpellation de son auditoire (Vargas, Magnin, Pradas, Manuel, Golovkine) et la scansion de ses phrases:

Vargas approuvait, inclinant la tête en même temps que Garcia inclinait la pipe. [...] Garcia pointa sur tous deux (Magnin et Vargas) le tuyau de sa pipe comme le canon d'un revolver [...]. Il remit sa pipe dans sa poche, et dit avec tristesse : 'Notre modeste fonction, monsieur Magnin, c'est d'organiser l'Apocalypse...' (Ibid., pp.608-610) ; Il (Garcia) leva le tuyau de sa pipe. 'Rien n'est plus difficile que de faire penser les gens sur ce qu'ils vont faire'. (Ibid., p.610)

L'auteur montre donc que la pipe donne certes du poids et de la matérialité au discours de Garcia mais aussi sert à combler ses failles et dresse un portrait caricatural et stéréotypé de l'intellectuel prédicateur, ce qui catalogue son point de vue. Garcia apparaît, à l'image du Négus et Pradas qui représentent les prototypes respectifs de l'anarchiste et du communiste, comme le symbole de l'intellectuel, ce qui en fait un personnage à la vision aussi sectaire et étriquée que les deux premiers, même si par ailleurs l'auteur lui confère des attributs de narrateur tels que le renseignement, la lecture, la traduction, l'interprétation ou l'organisation de la focalisation interne. Mais l'auteur, malgré ses intrusions, ne se situe pas dans la même logique que le narrateur omniscient car ses interventions sont brèves et se rapportent toutes au discours du narrateur-personnage: Garcia.

La focalisation interne reste de mise et l'auteur se contente d'ajouter la communication entre l'auteur et le lecteur à l'échange des points de vue entre les personnages réflecteurs. Dans L'espoir, on constate donc une tendance au partage de la voix et de la vision narrative entre les personnages eux-mêmes, entre les personnages et 
le narrateur ou entre les personnages et l'auteur. Mais toujours est-il que la focalisation interne est le procédé narratif principal suivant lequel ce roman est raconté. Ce qui donne plus de mouvement et de variété au récit, permet de relativiser et de confronter les points de vue et empêche la tentation de la prédication propre à la narration omnisciente. Cette limitation de la perspective et cette discrétion générale du narrateur rapprochent les récits malruciens du modèle de la communication théâtrale où toutes les perspectives se situent sur le même plan et, de surcroît, les ouvrent à une pluralité d'interprétations.

\section{CONCLUSION}

La narration de L'espoir partage avec celle de La condition humaine l'emploi généralisé de la focalisation interne. Mais elle s'en différencie par le fait qu'elle pousse l'éclatement des points de vue à l'extrême et qu'elle s'appuie non pas sur des points de vue individuels mais plutôt sur des points de vue collectifs. Les porteurs de point de vue ne sont plus des individus comme Kyo ou Clappique mais des groupes idéologiques comme ceux des communistes (Manuel, Pradas, Enrique) ou des anarchistes-libéraux (Hernández, Puig, Le Négus). Et l'antagonisme narratif ne se situe plus entre deux camps opposés mais à l'intérieur du même camp républicain entre les différentes tendances politiques (les anarchistes, les libéraux, les socialistes, les communistes, les intellectuels), d'autant que le point de vue fasciste est assez marginal.

Donc la généralisation de la focalisation sur les personnages et le remplacement du narrateur par deux types de personnages (Garcia et la nouvelle voix) rejoignent le théâtre dans la mesure où ils font écho à l'égalité des points de vue des personnages et à l'intrusion d'une 'voix off' au théâtre. La narration, dans L'espoir, conduit donc à la dramatisation du récit par à la fois la préservation du suspense de l'histoire et par la proscription d'une conclusion univoque. Cette implication de l'auteur nous ouvre la possibilité de nous intéresser à la fois aux points d'entrée et de sortie du texte romanesque, où l'auteur et le lecteur prennent directement ou indirectement contact ou inversement se séparent, et à la composition sous forme de scènes et d'actes des romans de Malraux.

\section{BIBLIOGRAPHIE}

Carrard, Philippe. (1976): Malraux ou le récit hybride. Essai sur les techniques narratives dans L'espoir, Paris, Lettres Modernes-Minard.

André Malraux. (1976): L'espoir in Romans, Paris, Gallimard, Bibl. de la Pléiade.

Robert S. Thornberry. (1977): André Malraux et l'Espagne, Genève, Droz.

Maurice Blanchot. (1959): «La douleur du dialogue», in Le Livre à venir, Paris, Gallimard, pp.207-218.

Jean-Pierre Blin. (1989): «L'aphorisme dans les romans» in Europe, n727-728, pp.93103. 
Francesca Cabasino. (Mars 1998): «Le mythe révolutionnaire entre dialogue et monologue dans L'espoir d'André Malraux» in Mots, n54, pp.58-59.

Jean Carduner. (Mars -Avril 1981): «L'espoir ou la fin de l'imaginaire de roman » in Revue d'histoire littéraire de la France, n², pp.236-247.

Monique Gosselin. (193): «Le rêve malrucien de l'intellectuel 'chaman'» in L'intellectuel et ses miroirs romanesques (1920-1960), Presses Universitaires de Lille, pp.97-117. 